\title{
Mainstreaming Adaptation in Development
}

\author{
Saleemul Huq and Hannah Reid
}

\section{Introduction}

Adaptation to climate change is one of two principal response strategies to the problem of humaninduced climate change (the other response strategy is "mitigation", which is to reduce the emissions of greenhouse gases, or GHGs). The principle difference between these two response strategies is that mitigation attempts to prevent the climate change problem from occurring at all (or getting worse), whilst adaptation aims to cope with the problem of climate impacts when they occur. This article describes how adaptation issues have been addressed in the climate negotiations to date. Because the impacts of climate change are likely to increase in the coming years and there is growing realisation that vulnerable countries and communities will be disproportionately adversely affected, much more attention is now being paid to adaptation than was previously the case, particularly by development organisations (see also Agrawala and Pachauri, this Bulletin). This article describes what is meant by adaptation, before focusing in more detail on the challenges facing the international community as it tries to gear up to responding to climate change. Specific actions on how adaptation can be mainstreamed in development, including financial issues, are provided in the concluding section.

\section{Mitigation vs. adaptation: a brief history}

As described in more detail by Professor Rogers and by Agrawala (this Bulletin), historically, the climate change issue has been seen primarily as one which is likely to occur in a gradual fashion in the medium- to long-term future (i.e. anything from 50 to 100 years' time) and that the policy priority in the short term (i.e. the next 10 to 20 years) has been the reduction of the emission of greenhouse gases (GHGs) through mitigation actions. Hence, the first decade of the negotiations under the United
Nations Framework Convention on Climate Change (UNFCCC) focused largely on the elaborating mitigation commitments and related institutional and financial mechanisms, to ensure that the main emitting countries took actions to reduce their emissions. This focus, which fitted well with early understandings of climate science and was fully in accordance with the precautionary principle set out in the Convention, tended however, to overshadow policy discussions about adaptation. Although both the UNFCCC and the Kyoto Protocol contain adaptation provisions, included largely at the insistence of vulnerable developing countries, the main emphasis of national and international climate policy has been on mitigation. This is reflected in the national communications (reports) submitted by developed and developing country Parties to the UNFCCC, which contain much less information about adaptation than about mitigation.

The publication of the Third Assessment Report of the Intergovernmental Panel on Climate Change (IPCC) in 2001 (IPCC 2001) highlighted that emissions continue to rise and that some impacts may already be discernible. It thus emphasised the need to pay more attention to adaptation responses in addition to mitigation responses. Accordingly, negotiations under the UNFCCC since then, particularly those at the seventh Conference of Parties (COP 7) held in November 2001 in Marrakech, Morocco (UNFCCC 2001), have paid much greater attention to adaptation issues, including agreement to establish new funds to assist poorer developing countries in dealing with the potential impacts of climate change. Because adaptation strategies to climate change, especially for the most vulnerable developing countries, are intimately linked to the larger development strategies in those countries, more development agencies have become active in the climate change regime. The linkages between adaptation to climate change and development, as well as the funding of adaptation in developing 
countries, including the role of donors and other players active the development, is explored further below and by Agrawala in this Bulletin.

\section{What is adaptation and adaptive capacity?}

Adaptation is the process of coping with the potential impacts of climate change. It can be characterised in different ways:

1. Anticipatory adaptation vs. reactive adaptation: Anticipatory adaptations are ones which are taken in anticipation of expected climate change impacts. Reactive adaptation occurs after the impacts have taken place.

2. Adaptation to climate change vs. adaptation to climate variability: The former refers to adaptation to anticipate human induced climate change, whereas the latter refers to adaptation to naturally occurring climate variability. In practice, there is little difference between actions that would enhance adaptation to climate change and actions that would enhance adaptation to climate variability, but the distinction is significant in the context of funding for adaptation under the UNFCCC (which is supposed to fund the former but not the latter).

Our understanding of adaptation depends on where society places its values. For example, ecologists argue that any definition of adaptation must also take into account species and communities other than humans, which must also adapt to climate change. This is important when it comes to prioritising adaptation activities and funding, as decisions on whether it is more important to protect unique ecosystems or focus on potentially catastrophic abrupt changes, such as the collapse of the Gulf Stream, will need to be made. The question of what degree of climate change is "dangerous" is dependent on these valuebased judgements, but science and local contextualised knowledge, such as that held by local communities, can also help inform the basis for such decision making.

Issues relating to equity and justice in the field of adaptation are also relevant and have attracted recent interest (Adger et al. forthcoming). Justice issues are important at several levels, such as the international legal framework, national adaptation policies and actions and everyday adaptation actions. At each level it is important to consider both distributive and procedural justice. For example, distributive justice is paramount when considering the funds for adaptation mentioned above and responsibility for climate change impacts, which in turn raise procedural issues about who decides these questions and on the basis of what information: is it donor-driven or country-led? Is it conditional on submissions of recent kinds of reports (such as national communications due under the UNFCCC) or dependent on ensuring there is sufficient information to ensure that resources are being well utilised? Procedural justice is also important when considering what kind of public consultation is necessary and possible in developing National Adaptation Programmes of Action (NAPAs) in the least developed countries (Paavola presentation, in Reid et al. 2004).

Adaptive capacity is the ability of a community (or country) to adapt to climate change. One key distinction is the difference between generic adaptive capacity and specific adaptation: generic adaptive capacity refers to the inherent or existing capacity of a community or country as a whole to cope with climate impacts. This is a function of levels of income, education, development, etc. of the whole community or country. Specific adaptive capacity refers to the capacity of the community or country to cope with the impacts of climate change based on an understanding of the anticipated impacts of human-induced climate change.

Two approaches have been used to try to understand adaptive capacity: initially a scenario, or top-down, approach was used to help understand how dangerous climate change is, and more recently a systems, or bottom-up, approach (Figure 1) has been used to understand how best to adapt to climate change. This approach assesses current vulnerabilities to climate change, starting at the community level, and is therefore the most appropriate approach to use when trying to identify current and future local risks to climate change.

\section{Adaptation and development linkages}

Adaptation to climate change is fundamentally linked to development both for the developed as well as the developing countries (both of which will be impacted by the adverse consequences of climate change). However, it is in the context of the developing countries that potentially adverse impacts 
Figure 1: Systems approach - vulnerabilities

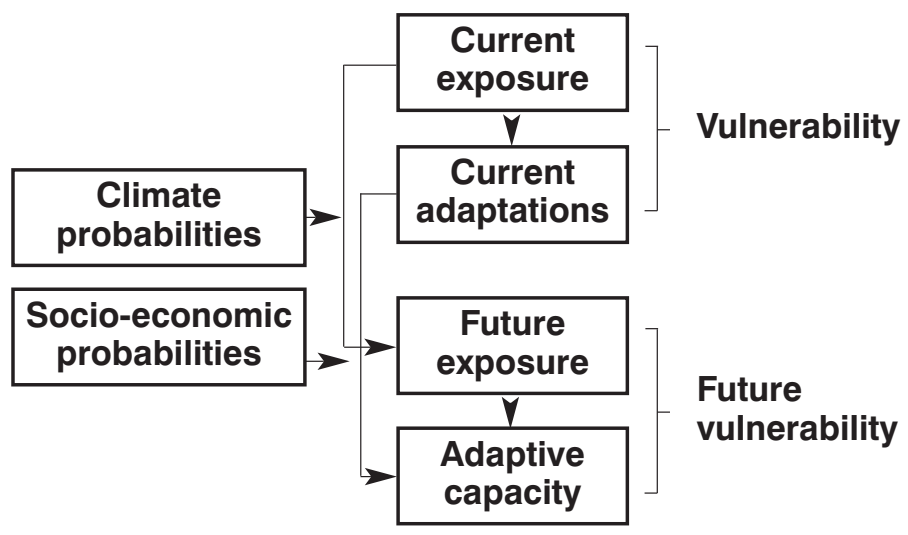

Source: Barry Smit presentation, in Reid et al. (2004).

of climate change will negatively affect development in a number of key sectors, including water resources, floods, droughts, agriculture and costal zone management. Linkages between adaptation and development occur at several different scales or levels and many are discussed in more detail by other contributors to this Bulletin as indicated below:

1. Local level: The most severely impacted communities in developing countries will be those communities living in geographic regions most exposed to climatic impacts (e.g. floodprone and drought-prone areas). As these people are generally poorer than the rest of the population within the country, they need to be targeted with programmes providing support for adaptation to climate change (see Pachauri and Agrawala, this Bulletin).

2. Sectoral level: Within countries, the most adversely impacted sectors include agriculture, water resource management, costal zone management as well as disaster (e.g. floods, cyclones and droughts) management (for more detailed discussion of particular sectors, see the contributions by Burton and May, Denton, Leach and Leach in this Bulletin). Policy makers, planners and managers in those sectors need to anticipate the future impacts of climate change in their sectoral planning.

3. National level: At the national level, policy makers will need to take into account potentially adverse impacts of climate change in different sectors and also take policy decisions across different sectors. One important feature of national policy making includes the need to address existing policies (and actions), which enhance (rather than reduce) vulnerabilities to climate change, and remove "maladaptations" to climate change.

4. Regional level: Many climate change impacts will be felt most acutely at the regional level in areas such as West Africa, eastern Africa, southern Africa and South Asia. Regional-level actions (e.g. for river basins or major drought prone areas such as the Sahel, see Devereux and Edwards, this Bulletin) may therefore be most appropriate. The regional level is also the smallest scale (at least at present) at which potential climate change impacts under different scenarios can be effectively modelled.

5. Global level: Actions implemented at the global level will require the global community of nations to act together under the UNFCCC as well as under other development oriented efforts. For example, reaching many of the Millennium Development Goals (MDGs) may be more difficult due to the adverse impacts of climate change. International cooperation will also be required for the development of innovative financial mechanisms such as insurance (see Hamilton, this Bulletin) and/or the more effective implementation of existing multilateral and bilateral sources of funding, discussed below. 


\section{Funding adaptation}

At present, most international funding is largely directed towards mitigation efforts, despite the clear need in many countries for concrete adaptation projects (see Greene, this Bulletin). Increasing international interest in adaptation has not been associated with equivalent increases in quantities of funding, reflecting a broader stagnation or actual decline of Official Development Assistance (ODA) to developing countries over the 1990s (see Bezanson, this Bulletin). However, several new funds dealing with adaptation were established following COP 7 (Huq 2002):

1. The Least Developed Countries Fund (LDCF) to enable the least developed countries (LDCs) to conduct National Adaptation Programmes of Action (NAPAs) to identify priority adaptation actions for further funding.

2. The Special Climate Change Fund (SCCF) for all developing countries, to support adaptation as well as other actions (e.g. including mitigation and technology transfer).

3. The Adaptation Fund (AF) under the Kyoto Protocol, which is to be based on contributions from the "Adaptation levy" placed on all transactions under the Clean Development Mechanism (CDM) of the Kyoto Protocol. This fund is meant to support "concrete adaptation" actions.

All three funds are to support adaptation in developing countries, but differ in important ways. The LDCF and SCCF are established under the UNFCCC and based on voluntary contributions from donor countries. The LDCF has already received funding (around US\$20 million) from a number of countries to enable the LDCs to carry out their respective NAPAs whereas the SCCF has not received any contributions yet. Despite this, a number of developed countries have made a "political commitment" to provide up to US\$400 million a year for adaptation activities in general, starting from 2005. The AF does not become operational until the Kyoto Protocol enters into force although CDM transactions are now being registered under the legal auspices of the UNFCCC and thus will start generating funding for the Fund once the Executive Board of the CDM starts issuing "certified emissions reductions" (on the CDM see Humphrey, this Bulletin).
In addition to these funds, the Global Environment Facility (GEF) has recently allocated US $\$ 50$ million to a new pilot adaptation action programme, for use over the next few years. Guidance on how these funds should be distributed is being developed. In the past, the GEF funded a wide range of initiatives, but now it has a more strategic approach towards funding adaptation activities; critical policy and project needs will now be identified using the Adaptation Policy Framework (UNDP 2003), UNFCCC national communications and the NAPAs.

The respective roles of the different sources of international funding support for adaptation actions are shown schematically in Figure 2. It is important to note that the GEF Trust funds (with the exception of its small grants programme aimed at small communities) are currently restricted to those projects that cross the barrier of funding by producing "global environmental benefits" - thus only addressing the "tip of the iceberg" - whereas the vast majority of the adaptation actions that will be needed will not be funded by the GEF because they generate co-benefits with both development as well as with adaptation to climate variability and not just adaptation to anthropogenically induced climate change alone. For example, the six case studies on adaptation to climate change undertaken under the auspices of the Organisation for Economic Cooperation and Development (OECD) (see Agrawala, this Bulletin) define adaptation to climate change narrowly so that it refers to only those climate change impacts that are deemed to be directly attributable to human-induced climate change, rather than to adaptation to the broader range of impacts associated with "climate variability". A narrow definition of climate impacts would tend to then only produce a small range of adaptation responses as being necessary and hence requiring funding - in essence addressing only a very narrow set of examples of adaptationdevelopment linkages (i.e. the "tip of the iceberg" in Figure 2) and hence missing the much larger set of relevant adaptation-development linkages where there are additional co-benefits. Leaving aside such important definitional issues, it should also be noted that communities that are most vulnerable to climate change (which need the assistance the most) are generally poorly served by existing mechanisms of funding (both international as well as national), because community-based projects tend to be 


\section{Figure 2: The Adaptation Funding "iceberg"}

International funding sources
Adaptation actors in developing countries

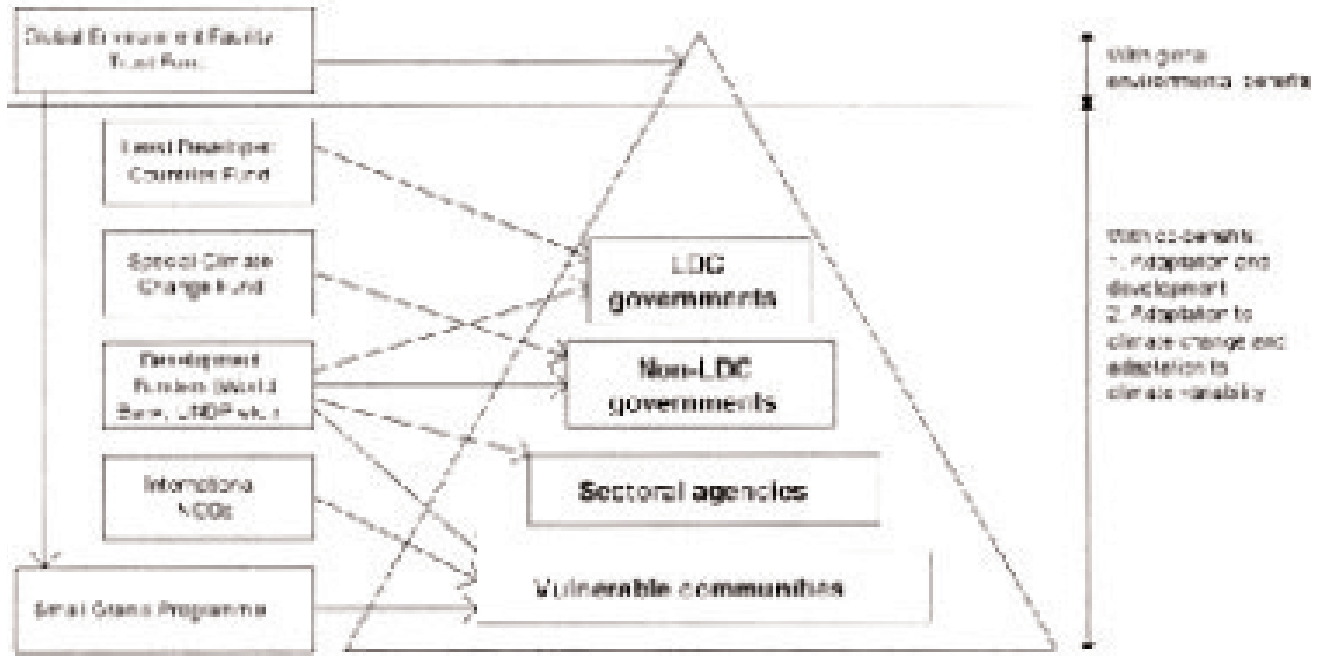

smaller, site-specific and thus less replicable which makes them unattractive to many funding agencies.

\section{Mainstreaming adaptation}

It is clear from the above that many of the activities that need to be undertaken to reduce vulnerability to the adverse impacts of climate change in developing countries relate closely to ongoing mainstream development activities at local, sectoral and national levels. Successful adaptation to climate change therefore requires incorporation of potential climate change impacts into ongoing strategies and plans at sectoral and national levels (Huq and Burton 2003; Huq et al. 2003, Agrawala, this Bulletin). This is best illustrated by taking a sector such as water resource management, where planners and managers are used to planning water management (e.g. for reservoirs) and will now have to plan for a future hydrological regime which includes potential climate change impacts (in other words they can no longer assume that the hydrological regimes of the past will be the same as future regimes - as illustrated by the example provided by Burton and May, this Bulletin). Once the relevant planners and managers have been provided with the appropriate methodologies and tools, they are quite capable of incorporating climate change issues into their regular planning (at relatively low costs). However, the issue of "mainstreaming" adaptation has caused some friction between stakeholders in climate change discussions to date. This is because the term "mainstreaming adaptation" is being used in the discussions in two distinct senses, namely:

1. Mainstreaming adaptation into development: This requires the main actors engaged in development work (governments, international development funding agencies, non-governmental organisations (NGOs), local communities, etc.) to increase their awareness of the potentially adverse impacts of climate change and then to "mainstream" issues relating to this into their regular activities.

2. Mainstreaming adaptation funding: Broadly speaking this refers to utilisation of existing ODA resources to fund climate change related adaptation.

The second sense of mainstreaming adaptation causes friction for two reasons. First, it is problematic because it blurs funding mandated by the Convention (which is supposed to be "new and additional") with funding donors are supposed to provide through 
their "regular" ODA for developmental activities generally (i.e. development activities that do not factor in climate change). Second, the use of existing ODA is being proposed because of the restricted approach to funding adopted by the Global Environment Facility (GEF) which operates the Convention's financial mechanism, including the new funds outlined above. To date, the extent to which the GEF has been able to fund climate-related adaptation has been extremely limited because of its "incremental cost" approach which allows it to only fund the component of adaptation projects that produces "global environmental benefits". In the case of mitigation projects, calculating global benefits is conceptually quite straightforward as one less ton of GHG emissions is a global benefit and the costs of avoiding it can be calculated relatively clearly. However, in the case of adaptation projects such a calculation is much more difficult as most of the benefits of adaptation activities accrue locally rather than globally. Additionally trying to separate and then calculate the costs of adaptation to climate change (as opposed to climate variability) is almost impossible as discussed above. Use of ODA to fund climate adaptation would avoid the procedural changes that are necessary for the GEF to ensure the existing and new Marrakesh funds are operationalised in a flexible manner to provide funding for a wide range of adaptation activity, leaving other sources of ODA to support activities that are solely (or primarily) for ongoing development goals such as education, sanitation, health and poverty reduction.

\section{What next?}

The importance of adaptation as a response action to the problem of human-induced climate change has recently become increasingly well recognised both in the climate change discussions (e.g. the Delhi Declaration at COP 8) as well as within (at least some of) the development funding agencies, for example the ten funding agencies who prepared the report on climate change and poverty (Sperling 2003). However, the problem has received little attention from most developing countries (with the exception of the small island states) or from the research and NGO community. Efforts therefore need to be enhanced to raise the level of awareness of climate change impacts (and hence the need for adaptation) amongst all relevant stakeholder groups, whilst simultaneously supporting adaptation actions on the ground, targeting the most vulnerable countries and communities. Specific actions that need to be taken by different stakeholder groups include the following:

1. UNFCCC: To agree pragmatic rules and criteria for supporting adaptation actions in developing countries under the newly created adaptation funds (primarily the LDCF and SCCF).

2. Developed countries: A number of developed countries (including the European Union, Canada and Norway) have already provided a relatively small amount of funding for the LDCF, but none have committed any funds for the SCCF yet. They need to do so (at least to the level of their "political commitment" at COP 6bis to provide US $\$ 400$ million/year). Incorporating climate adaptation into development will require additional financial resources.

3. GEF: The US $\$ 50$ million allocated by the GEF to support adaptation activities needs to be used to support adaptation actions (rather than just studies as has occurred in the past). However, current GEF rules on incremental costs for global benefits provide a major barrier to funding adaptation projects. The GEF also favours larger projects, which does not necessarily respond to the needs and scale of the most vulnerable countries and communities. These barriers need to be overcome.

4. Other bilateral/multilateral donors: The inherent unreplicability of adaptation projects (each will need to be site/community-specific) and the fact many are likely to be small-scale (if they are to enhance local livelihoods) is a major challenge for international donors, and may require institutions to become more responsive and flexible to these realities.

5. LDCs: They need to complete their respective NAPAs as soon as possible (and in a participatory manner with inputs from civil society and the most vulnerable communities) and determine priority actions.

6. Other developing countries: All the other developing countries also need to conduct adaptation planning to identify key adaptation actions. This can be done under the aegis of preparing their second National Communications and could utilise tools such as the Adaptation Policy Framework (UNDP 2003).

7. Development NGOs: International and national NGOs working in the development sector have so far engaged little with the problem of climate 
change and the potentially adverse impacts it will have on their target groups (who are often the most vulnerable communities) (Reid et al. 2004).

8. Researchers: The level of research on adaptation to climate change has been relatively poor to date, particularly when compared with the level of research effort invested in mitigation. More activists and researchers need to be persuaded to examine adaptation to climate change and to answer questions such as: what are the indictors and components of adaptive capacity and how can they best be strengthened? What are good tools for adaptation in the different sectors (e.g. agriculture, water resources management, costal zone management, etc.)? What links between adaptation and mitigation should be developed?

There are many theoretical, policy and practical challenges ahead. Ensuring there is greater

\section{References}

Adger, W.N., Huq, S., Mace, M.J. and Paavola, J. (eds), forthcoming 2005, Fairness in Adaptation to Climate Change, Cambridge, MA: The MIT Press

Huq, S., 2002, 'The Bonn-Marrakech agreements on funding', Climate Policy, Vol 2 Nos 2-3: 243-6

Huq, S. and Burton, I., 2003, 'Funding adaptation to climate change: what, who and how to fund?', Sustainable Development Opinion Paper, London: IIED

Huq, S., Rahman, A., Konate, M., Sokona, Y. and Reid, H., 2003, Mainstreaming Adaptation to Climate Change in Least Developed Countries (LDCs), London: IIED

IPCC, 2001, 'Climate Change 2001: Impacts, Adaptation, and Vulnerability', a contribution awareness of climate change by the development community - and vice versa - will thus require more concerted collaborative efforts bringing together a global network of donors, policy makers, researchers and those working at the local level. Because our knowledge of climate impacts and adaptive capacity will evolve, and because surprises and shocks will occur, such networks must necessarily be of a long-term nature if continuous learning and dialogue is to take place at multiple levels on adaptation issues. Unfortunately, the funding cycles of most donors, research and policy bodies do not currently favour this kind of continuous large-scale effort, which has to bring together those engaged in theoretical, policy and implementation work from literally all corners of the globe. A first step in right direction would be the creation of mechanisms and channels to facilitate such learning and dialogue.

of the Working Group II to the Third Assessment Report of the Intergovernmental Panel on Climate Change (J.J. McCarthy, O.F. Canziani, N.A. Leary, D.J. Dokken and K.S. White, eds), Cambridge: Cambridge University Press

Reid, H., Huq, S. and Murray, L., 2004, Adaptation Day at COP 9, London: IIED

Sperling, F. (ed.), Multi-Agency Report, 2003, Poverty and Climate Change: Reducing the Vulnerability of the Poor through Adaptation, Washington, D.C.: The World Bank

UNDP, 2003, The Adaptation Policy Framework, New York: UNDP-GEF

UNFCCC, 2001, The Marrakech Accords and the Marrakech Declaration, FCCC/CP/2001/13 and Addendum 1-4 